\title{
Winds and Outflows in Starburst Galaxies and AGN
}

\author{
Stefanie Komossa, Günther Hasinger \\ Max-Planck-Institut für extraterrestrische Physik, Giessenbachstr., \\ D-85748 Garching, Germany \\ Hartmut Schulz ${ }^{\dagger}$ \\ Ruhr-Universität Bochum, Astronomisches Institut, 44780 Bochum, \\ Germany \\ ${ }^{\dagger}$ passed away in August 2003
}

\begin{abstract}
Winds and outflows in starburst galaxies and AGN provide important information on the physics of the 'central engine', the presence and evolution of (nuclear) starbursts, and the metal enrichment of the nuclear environment and the intergalactic medium. Here, we concentrate on two examples, X-ray observations of the (U)LIRG NGC 6240 and the BAL quasar APM 08279+5255.
\end{abstract}

\section{Introduction}

We present recent results on winds and outflows in starburst galaxies and active galactic nuclei (AGN) based on observations carried out with the Chandra X-ray observatory.

Outflows play a crucial role in enriching the nuclear environment with matter from the core region, carrying metals and possibly dust. They thus represent an important component when studying the recycling of interstellar and intergalactic matter.

In starburst galaxies, superwinds are driven by nuclear starbursts. Their study provides important information on the evolution of these winds, the metal content they carry, and the process of IGM enrichment with metals and/or dust.

In AGN, outflows may be driven by radiation pressure of the central continuum source. Components in outflow include possible accretion-disk winds and ionized absorbers. Chandra and XMM-Newton spectra of AGN with warm absorbers are rich in absorption features which allow study of the ionized material, its origin, evolution, and interaction with the environment, in great detail (e.g., Simkin \& Roychowdhury 2002).

\section{Starburst-driven Superwinds, AGN environment: NGC 6240}

\subsection{The galaxy NGC 6240}

Ultraluminous infrared galaxies (ULIRGs) are characterized by their huge luminosity output in the infrared, predominantly powered by super-starbursts and/or 
hidden AGN (e.g., Genzel et al. 1998, Sanders et al. 1988, 1999). Many distant SCUBA sources, massive and dusty galaxies, are believed to be ULIRG equivalents at high redshift. Local ULIRGs are therefore ideally suited to study the physics of galaxy formation and evolution (many ULIRGs are mergers), the processes of IGM enrichment, the physics of superwinds driven by the nuclear starbursts, and to assess the frequency of hidden AGN at their cores. The questions regarding the onset of starburst and AGN activity and their evolution in mergers are of fundamental importance for our understanding of AGN/black hole formation and evolution in general.

Here, we would like to concentrate on the galaxy NGC 6240, one of the nearest members of the class of (U)LIRGs, considered to be a key representative of its class. The galaxy is a merger in the process of forming an elliptical galaxy.

ROSAT observations (Schulz et al. 1998) showed that NGC 6240 is special in its highly luminous extended X-ray emission, making it one of the most luminous X-ray emitters in extended emission known among field galaxies (Komossa et al. 1998).

\subsection{Chandra results}

Given the complex nature of the X-ray emission of NGC 6240 with contributions from many components suggested from previous X-ray observations, spatially resolved spectroscopy is crucial to disentangle all contributing components, determine their nature, and derive their physical properties. Here, we report results from the first spatially resolved X-ray spectroscopy of NGC 6240, carried out with the ACIS-S instrument onboard the Chandra X-ray observatory (see also Komossa et al. 2003).

The Chandra image of NGC 6240 reveals a wealth of structure, changing in dependence on energy. A large part of the X-ray emission of NGC6240 is extended, confirming previous results first obtained with the ROSAT instruments.

Basically, the extended emission of NGC 6240 shows three components: firstly, some weak, widely extended halo emission, which was already hinted for by our previous ROSAT observations, not discussed further here. Secondly, an emission component spatially coincident with the $\mathrm{H} \alpha$ emission of NGC 6240 . Thirdly, a more compact, harder, extended component, likely related to some nuclear starburst activity.

In the Chandra ACIS-S image, several X-ray 'loops' and knots are visible which correlate well with the $\mathrm{H} \alpha$ emission of NGC 6240 (Fig. 2), and which are most prominent between 1 and $2.5 \mathrm{keV}$. Above $1.5 \mathrm{keV}$, X-ray emission from the direction of the northern nucleus of NGC 6240 starts to emerge. The hard X-ray image is dominated by emission from two compact sources, spatially coincident within the errors with the IR positions of the two nuclei of NGC 6240.

The extended X-ray emission of NGC 6240 of "butterfly-like" shape and coincident with the $\mathrm{H} \alpha$ emission, is generally well described by a MEKAL model with $k T \simeq 0.8 \mathrm{keV}$ and absorption with a column density $N_{\mathrm{H}} \simeq 310^{21} \mathrm{~cm}^{-2}$. Abundances are not well constrained, but fits typically yield $Z \approx 0.1$ solar when applying simple one-component thermal models.

In addition to the widely extended emission, a more compact nuclear component, still extended, is present. Representative of this emission, we have determined the spectrum of an emission-blob south-west of the Southern nucleus 
of NGC 6240. It is well fit by a MEKAL model of temperature $k T \simeq 3 k e V$ and abundances of $Z \approx$ solar.

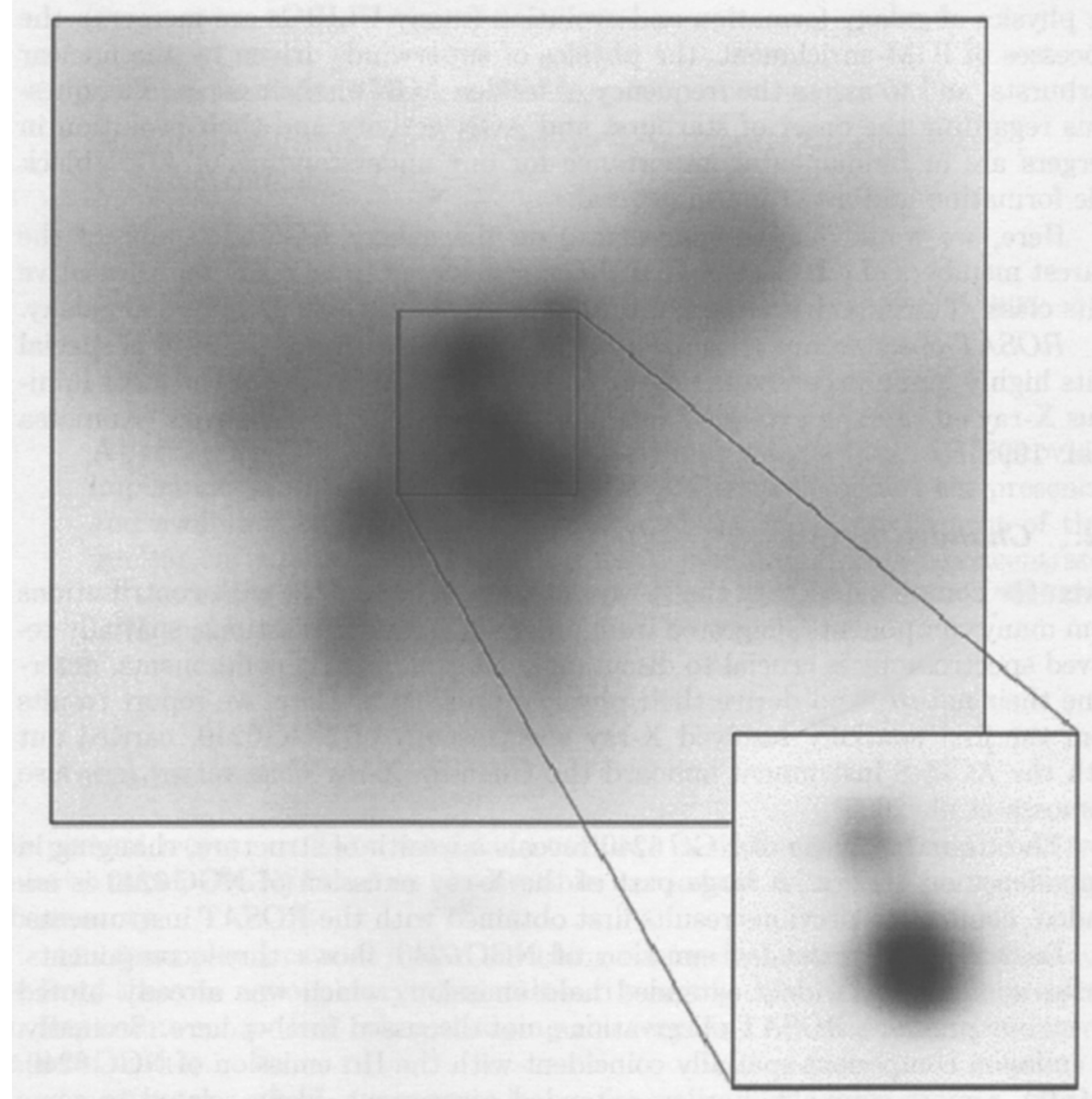

Figure 1. X-ray energy image of NGC 6240 . The inset zooms onto the two hard nuclei.

The trend of decreasing abundances with increasing nuclear separation of the starburst component of Mrk 273 was noted by Xia et al. (2002). Whether this trend is true for NGC 6240, or whether the lower abundances inferred for the more extended starburst component of NGC 6240 are still an artifact of not applying more complex emission models (e.g., Komossa et al. 2000), has yet to be determined. It would require much deeper Chandra observations of NGC 6240 (and other (U)LIRGs) than presently available, in combination with more complex, possibly non-equilibrium emission models.

According to superwind models of Schulz et al. (1998), a mechanical input power of $L_{\text {mech }}=310^{43} \mathrm{erg} / \mathrm{s}$ (derived from a SN-rate of $3 /$ year) can, within $310^{7} \mathrm{yrs}$, drive a single shell to an extent of $R \sim 10 \mathrm{kpc}$ within a medium of 0.1 
$\mathrm{cm}^{-3}$ particle density. This model reproduces the observed X-ray luminosity of the extended, butter-fly shaped, starburst-related X-ray emission of NGC 6240 of $\sim 10^{42} \mathrm{erg} / \mathrm{s}$.

The spectra of both X-ray nuclei of NGC 6240 are very hard in X-rays and show strong, $\sim$ neutral iron $\mathrm{K} \alpha$ lines. These properties identify both nuclei of NGC 6240 as active, and suggest a scattering geometry for both AGN in NGC 6240 (the core components are discussed in greater detail by Komossa et al. 2003). In addition to the $6.4 \mathrm{keV}$ iron line, which is traced back to fluorescence from cold material in the AGN environment, a weaker line from highly ionized iron shows up, which could be related to supernova remnants or an ionized scatterer (see Komossa et al. 1998 for models of a warm scatterer in NGC 6240, and Boller et al. 2003 for recent XMM data which resolved the high-ionization iron-line complex in two components.)

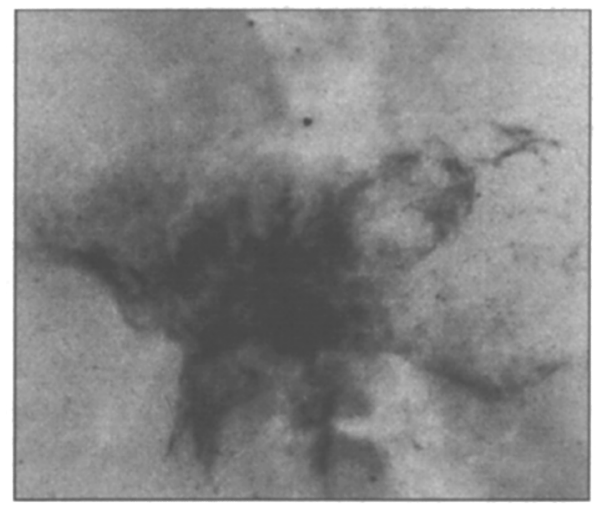

HUBgLe DPTICAL

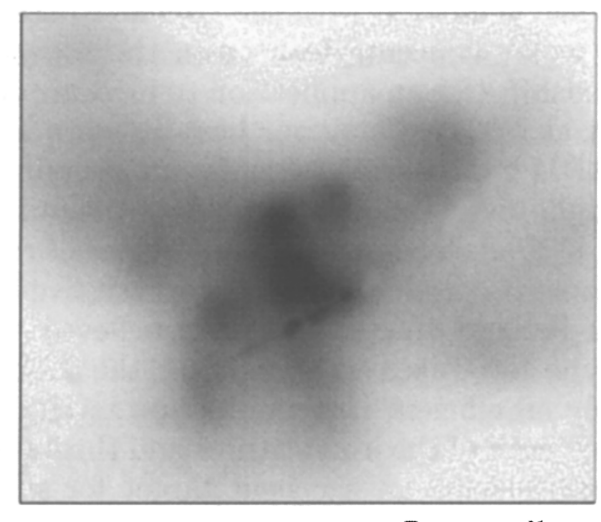

CHANDRA X-RAY

Figure 2. Comparison of the $\mathrm{H} \alpha$ image (Gerssen et al. 2001) with the X-ray image of NGC 6240 [NASA/CXC/Komossa et al. 2003].

\section{Absorption and outflows in AGN}

Cold and 'warm' absorption is ubiquitous in the AGN environment. X-ray absorption and emission features provide valuable diagnostics of the physical conditions in the X-ray gas and, in particular, allow to measure elemental abundances at high redshift, with profound consequences for our understanding of the star formation history in the early universe.

\subsection{BAL quasars}

Here, we would like to concentrate on BAL quasars. They are characterized by broad UV absorption lines. It has been suggested that these lines arise in a flow of gas which rises vertically from a narrow range of radii from the accretion disk. The flow then bends and forms a conical wind moving radially outwards (Elvis 2000). Variants of radiatively-driven disk-winds were explored (e.g., Proga et al. 2000, and references therein). In some of these models, an X-ray absorber 
shields the wind downstream from soft X-rays, allowing resonant line driving to remain effective and accelerate the outflowing BAL wind up to $\sim 0.1 \mathrm{c}$.

These high-velocity BAL winds may play a crucial role in metal enrichment of the interstellar medium of the galaxy, possibly even the IGM.

\subsection{The quasar APM $08279+5255$}

The high-redshift BAL quasar APM $08279+5255$ is magnified by a gravitational lens and is among the most luminous objects in the universe (even after correction for lensing). It has one of the best-measured X-ray spectra among BALs. The recent XMM-Newton observation led to the detection of a strong absorption feature of ionized iron, interpreted as K-edge, arising from a warm absorber of high column density (Hasinger et al. 2002), and variable on short timescales, when compared with the Chandra observation (Chartas et al. 2002).

We concentrate here on the aspect of deriving metal abundances at high redshift (for an application to measure cosmological parameters, see e.g. Alcaniz et al. 2003). Using our best-fit warm absorber model of the XMM spectrum of $\mathrm{APM} 08279+5255$, we infer an abundance of $\mathrm{Fe} / \mathrm{O} \simeq 3 \times$ solar which is rather high, given the high redshift of the object.

The element iron plays a special role in chemical enrichment scenarios, because its production is delayed relative to other elements (e.g., Fig. 1 of Hamann \& Ferland 1993), since it is believed to be mostly produced in supernovae of type Ia. Taken at face value, the XMM observation of APM 08279+5255 imply an efficient iron production mechanism in the early universe. The unusual strength of the iron features and the indications for strong variability make APM $08279+5255$ an excellent target for simultaneous, deep follow-up observations with Chandra and XMM-Newton.

While Fe X-ray features have only been observed in very few BALs so far, with future X-ray missions like XEUS (Arnaud et al. 1998) we will be able to observe more objects with high accuracy. The iron absorption lines and Kedges provide a unique probe of matter at high redshift because, firstly, they are easy to measure even, or, particularly, at high redshift, and secondly, $\mathrm{Fe}(/ \mathrm{O})$ abundance measurements in the early universe are important for constraints on the early star formation history.

Science issues that we will be able to address in detail with future X-ray missions for the first time, particularly at high redshift, include the determination of metal abundances of X-ray absorbers by detection of metal absorption edges, the determination of the composition of dust mixed with cold and ionized gas (K-edges of metals in cold dust and cold gas will be resolvable from each other for the first time), measurements of the velocity field of the gas, and the utilization of these results to investigate the effects of reprocessing of gas and dust in AGN from high to low redshift.

Acknowledgments. I, St.K., am grateful to Hartmut Schulz for intense collaborations on NGC 6240 ever since I started in astrophysics, for sharing his wide knowledge, and for ongoing discussions on many topics of astrophysics. Hartmut passed away in August 2003, after a short, severe illness.

We thank R. van der Marel for providing the HST H $\alpha$ image of NGC 6240. 


\section{References}

Alcaniz J.S., Lima, J.A.S., Cunha, J.V. 2003, MNRAS, 340, L39

Arnaud, M., et al. 1999, The XEUS Science Case, ESA

Boller, T., et al. 2003, A\&A, 411, 63

Chartas, G., Brandt, W.N., Gallagher, S.C., Garmire, G.P., 2002, ApJ, 597, 169

Elvis, M., 2000, ApJ, 545, 63

Genzel, R., Lutz, D., Sturm, E., et al. 1998, ApJ, 498, 579

Gerssen, J., van der Marel, A.P., Axon, D., Mihos, C., Hernquist, L., Barnes, J.E., 2001, ASP Conf. Ser., 249, 665

Hamann, F., Ferland, G.J. 1993, ApJ, 418, 11

Hasinger, G., Schartel, N., Komossa, S. 2002, ApJ, 573, L80

Komossa, S., Schulz, H., Greiner, J. 1998, A\&A, 334, 110

Komossa, S., Breitschwerdt, D., Böhringer, H., Meerschweinchen, J. 2000, Ap\&SS, 272,295

Komossa, S., Burwitz, V., Hasinger, G., Predehl, P., Kaastra, J.S., Ikebe, Y., 2003, ApJ, 582, L15

Proga, D., Stone, J.M., Kallman, T.R., 2000, ApJ, 543, 686

Sanders, D.B., Soifer, B.T., Elias, J.H., et al. 1988, ApJ, 325, 74

Sanders, D.B. 1999, Ap\&SS, 266, 331

Schulz, H., Komossa, S., Berghöfer T., Boer, B. 1998, A\&A, 330, 823

Simkin, M.V., Roychowdhury, V.P., 2002, cond-mat/212043

Xia, X.Y., Xue, S.J., Mao, S., et al. 2002, ApJ, 564, 196 